\section{REDIMAT}

Journal of Research in Mathematics Education
Hipatia Press

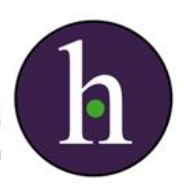

Instructions for authors, subscriptions and further details:

http://redimat.hipatiapress.com

\title{
Interpretación de Resúmenes Estadísticos por Futuros Profesores de Educación Secundaria
}

María M. Gea ${ }^{1}$, Carmen Batanero ${ }^{1}$, José A. Fernandes ${ }^{2}$ y Pedro Arteaga $^{1}$

1) Universidad de Granada, España

2) Universidade do Minho, Portugal

Fecha de publicación: 24 de Junio de 2016

Periodo de edición: Junio 2016-Octubre 2016

Para citar esta artículo: Gea, M.M., Batanero, C., Fernandes, J.A. \& Arteaga, P. (2016). Interpretación de resúmenes estadísticos por futuros profesores de educación secundaria. REDIMAT, 5(2), 135-157. doi: 10.4471/redimat.2016.1902

To link this article: http://dx.doi.org/10.4471/redimat.2016.1902

PLEASE SCROLL DOWN FOR ARTICLE

The terms and conditions of use are related to the Open Journal System and to Creative Commons Attribution License (CC-BY). 


\section{Interpretation of Summaries on Statistics Addressed to Prospective Teachers in Middle School}

María M. Gea

Universidad de Granada

José A. Fernandes

Universidade do Minho
Carmen Batanero

Universidad de Granada

Pedro Arteaga

Universidad de Granada

(Received: 20 January 2016; Accepted: 13 June 2016; Published: 24 June 2016)

\section{Abstract}

The aim of this work was assessing the interpretation of statistical summaries carried out by prospective secondary and high school teachers. We analyse the responses by 65 students in the Master of Teacher Education, specialty of Mathematics, to two tasks. In the first task they should interpret the mean, median, mode, minimum, maximum, quartiles and standard deviation in a data distribution; in the second task, they have to select the most representative average using the same statistical summaries and a box plot. The interpretation of statistics, the selected average and the participants' justifications were generally correct, and we obtained better results than in previous research with students and prospective primary school teachers. However, we observe some conflicts on the meaning of the interpreted statistics, which should be overcome to assure a good teaching performance in their professional future.

Keywords: Common content knowledge, prospective teachers, secondary and high school, statistical summaries 


\section{Interpretación de Resúmenes Estadísticos por Futuros Profesores de Educación Secundaria}

María M. Gea

Universidad de Granada

José A. Fernandes

Universidade do Minho
Carmen Batanero

Universidad de Granada

Pedro Arteaga

Universidad de Granada

(Recibido: 9 Noviembre 2015; Aceptado: 7 Febrero 2016; Publicado: 24 Junio 2016)

\section{Resumen}

El objetivo de este trabajo fue evaluar la interpretación de resúmenes estadísticos que realizan los futuros profesores de Educación Secundaria y Bachillerato. La evaluación se lleva a cabo analizando las respuestas de una muestra de 65 estudiantes del Máster de Formación del Profesorado, especialidad de Matemáticas, a dos tareas. En la primera se les pide interpretar la media, mediana, moda, mínimo, máximo, cuartiles y desviación típica de una distribución de datos y en la segunda, elegir un promedio representativo utilizando los anteriores estadísticos y un gráfico de caja y bigotes de la misma distribución. Las interpretaciones de los estadísticos, el promedio representativo elegido y el modo en que se justifica la elección fueron generalmente correctos, obteniéndose mejores resultados que en investigaciones previas con estudiantes y futuros profesores de educación primaria. Sin embargo, se observan conflictos sobre el significado de los estadísticos interpretados, que se deberían superar para lograr una buena labor docente de estos futuros profesores.

Palabras clave: Conocimiento común del contenido, futuros profesores, Educación Secundaria y Bachillerato, resúmenes estadísticos 
L

os resúmenes estadísticos sirven para caracterizar una distribución de datos y para comparar varias distribuciones entre sí. Su comprensión no se reduce a conocer su definición o fórmula o a usarlos como instrumento, sino que incluye reconocer su utilidad dentro de un contexto (Makar y Confrey, 2005). Estas competencias son parte de la alfabetización estadística, reclamada desde las instituciones educativas y los institutos de estadística, por su importancia en la toma de decisiones y la participación activa en la sociedad actual. Así por ejemplo, Wallman (1993) la define cómo:

Alfabetización estadística es la capacidad de comprender y evaluar críticamente los resultados estadísticos que impregnan nuestra vida cotidiana - junto con la capacidad de apreciar las contribuciones que el pensamiento estadístico puede hacer en las decisiones públicas y privadas, profesionales y personales (Wallman, 1993, p. 1).

Uno de los mecanismos principales para lograr la cultura estadística para todos ha sido la incorporación generalizada en el currículo de contenidos estadísticos. La enseñanza de las medidas de posición central y dispersión se incluye en España con diferente profundidad en los distintos niveles educativos, tanto en los decretos vigentes hasta la fecha (MEC, 2006; 2007a; 2007b), como en los nuevos decretos (MECD, 2014; 2015), cuyo contenido sobre el tema resumimos en la Tabla 1 . Se puede observar que algunos de estos resúmenes se introducen ya en la educación primaria en forma intuitiva, y en las etapas educativas posteriores se van formalizando progresivamente, tanto desde la estadística descriptiva, como desde la probabilidad e inferencia. Además de incluir los conceptos y su cálculo, el currículo enfatiza el uso e interpretación de estos estadísticos para asegurar su correcto aprendizaje. Por todo ello, será necesaria una comprensión profunda del tema por parte del futuro profesor de matemáticas.

La investigación sobre comprensión de estos resúmenes estadísticos es muy amplia (ver un resumen en Shaughnessy, 2007) y describe errores conceptuales, de interpretación y cálculo en alumnos de diferentes edades. Aunque su aparente sencillez puede sugerir que este es un tema trivial para los futuros profesores, la síntesis que realizan Jacobbe y Carvalho (2011) muestra que muchos estudiantes para profesor de educación primaria no alcanzan una compresión profunda. La investigación sobre el conocimiento del tema por parte de los futuros profesores de secundaria es muy escasa y se limita a su capacidad de cálculo o uso de los mismos. 


\section{María M. Gea et al. - Interpretación de Resúmenes Estadísticos}

\section{Tabla 1}

Contenidos relacionados con los resúmenes estadísticos en el currículo actual

\begin{tabular}{|c|c|c|}
\hline \multicolumn{2}{|l|}{ Curso } & Contenidos relacionados con los resúmenes estadísticos. \\
\hline \multicolumn{2}{|c|}{ Educación primaria } & $\begin{array}{l}\text { Iniciación intuitiva a las medidas de centralización: la } \\
\text { media aritmética, la moda y la mediana, así como al } \\
\text { rango, como medida de dispersión. }\end{array}$ \\
\hline \multicolumn{2}{|c|}{$1^{\circ}$ y $2^{\circ}$ de E.S.O. } & Medidas de tendencia central. Medidas de dispersión. \\
\hline \multirow[t]{2}{*}{$\begin{array}{l}3^{\circ} \mathrm{de} \\
\text { E.S.O. }\end{array}$} & $\begin{array}{l}\text { Enseñanzas } \\
\text { académicas }\end{array}$ & $\begin{array}{l}\text { Parámetros de posición. Cálculo, interpretación y } \\
\text { propiedades. Parámetros de dispersión. Diagrama de caja } \\
\text { y bigotes. Interpretación conjunta de la media y la } \\
\text { desviación típica. }\end{array}$ \\
\hline & $\begin{array}{l}\text { Enseñanzas } \\
\text { aplicadas }\end{array}$ & $\begin{array}{l}\text { [Igual que en Matemáticas orientadas a las enseñanzas } \\
\text { académicas y se añade:] Rango, recorrido intercuartílico } \\
\text { y desviación típica. Cálculo e interpretación. }\end{array}$ \\
\hline \multicolumn{2}{|c|}{$\begin{array}{l}4^{\circ} \text { de E.S.O. en } \\
\text { enseñanzas académicas y } \\
\text { aplicadas }\end{array}$} & $\begin{array}{l}\text { Medidas de centralización y dispersión: interpretación, } \\
\text { análisis y utilización. Comparación de distribuciones } \\
\text { mediante el uso conjunto de medidas de posición y } \\
\text { dispersión. }\end{array}$ \\
\hline \multicolumn{2}{|c|}{$\begin{array}{l}1^{\circ} \text { Bachillerato en } \\
\text { Ciencias Sociales }\end{array}$} & $\begin{array}{l}\text { Medias y desviaciones típicas marginales y } \\
\text { condicionadas. Media, varianza y desviación típica en las } \\
\text { distribuciones de probabilidad. }\end{array}$ \\
\hline \multicolumn{2}{|c|}{$\begin{array}{l}1^{\circ} \text { Bachillerato en } \\
\text { Ciencias }\end{array}$} & $\begin{array}{l}\text { Medias y desviaciones típicas marginales y } \\
\text { condicionadas. }\end{array}$ \\
\hline \multirow{4}{*}{\multicolumn{2}{|c|}{$\begin{array}{l}2^{\circ} \text { Bachillerato de } \\
\text { Ciencias } \\
2^{\circ} \text { Bachillerato Ciencias } \\
\text { Sociales }\end{array}$}} & Variables aleatorias discretas. Media, varianza y \\
\hline & & desviación típica. Tipificación de la distribución normal. \\
\hline & & Parámetros de una población y estadísticos obtenidos a \\
\hline & & $\begin{array}{l}\text { partir de una muestra. Media y desviación típica de la } \\
\text { distribución de la media muestral. Distribución de la } \\
\text { media muestral en una población normal. Intervalo de } \\
\text { confianza para la media poblacional de una distribución } \\
\text { normal con desviación típica conocida, así como para la } \\
\text { media poblacional de una distribución de modelo } \\
\text { desconocido en el caso de muestras grandes. }\end{array}$ \\
\hline
\end{tabular}


Los principales trabajos en esta línea son debidos a Batanero, Godino y Navas (1997), Canada (2004), Groth y Bergner (2006), Hammerman y Rubin (2004), Jacobbe (2008) y Makar y Confrey (2005). El objetivo de nuestro trabajo es completarla, evaluando las interpretaciones de las medidas de posición central y dispersión y su competencia para elegir un promedio representativo.

\section{Marco Teórico}

Shulman (1986) desglosó el conocimiento requerido por los profesores para abordar con éxito la enseñanza de la matemática en conocimiento del contenido, conocimiento del contenido pedagógico y conocimiento del currículo. Esta investigación promovió un gran número de investigaciones orientadas a la evaluación y desarrollo del mismo en los profesores de matemática (descritas, por ejemplo, en Blömeke, Hsieh, Kaiser y Schmidt, 2014; Even y Ball, 2009 y Llinares y Krainer, 2006).

Un marco teórico que ha tenido un gran impacto en la investigación sobre formación de profesores es el del conocimiento matemático para la enseñanza (MKT) (Adler y Venkat, 2014; Ball, Lubienski y Mewborn, 2001; Hill, Ball y Schilling, 2008). Su finalidad es caracterizar el conocimiento que necesita el profesor para enseñar matemáticas, y servir de aporte para investigar la relación entre enseñanza y aprendizaje de las matemáticas. Al igual que en el trabajo de Shulman (1986), se considera el conocimiento del contenido y el conocimiento del contenido pedagógico, dividiendo el primero en tres componentes:

- El conocimiento común del contenido, es el que posee una persona (no necesariamente el profesor) después de haber estudiado el tema; se trataría del conocimiento que el profesor ha de enseñar a sus alumnos.

- El conocimiento matemático avanzado (que los autores denominan conocimiento en el horizonte matemático), va más allá del conocimiento común; incluye conocimiento del tema a un nivel superior y las conexiones con otras materias. Sirve al profesor para relacionar un contenido particular con otros temas y diferentes áreas.

- El conocimiento especializado del contenido, es el aplicado por el profesor para articular tareas de enseñanza referidas al tema a enseñar. 


\section{María M. Gea et al. - Interpretación de Resúmenes Estadísticos}

En nuestro estudio analizamos únicamente el conocimiento común del contenido sobre los resúmenes estadísticos de valor central y dispersión, evaluándolo mediante las interpretaciones que los futuros profesores realizan de dichos resúmenes y los criterios que utilizan para elegir una medida de posición central. Aunque estos conocimientos serían avanzados en el futuro profesor de educación primaria (pues no se incluyen en este nivel educativo), los consideramos comunes en el futuro profesor de Educación Secundaria y Bachillerato, pues se incluyen en estos niveles educativos.

\section{Antecedentes}

Las principales investigaciones que han analizado el conocimiento de los profesores sobre los resúmenes estadísticos se resumen en Jacobbe y Carvalho (2011) y Ortiz y Font (2014). En nuestro trabajo utilizaremos como apoyo las de Batanero, Godino y Navas (1997), Groth y Bergner (2006), Hammerman y Rubin (2004) y Jacobbe (2008) sobre medidas de posición central y las de Canada (2004) y Makar y Confrey (2005), que analizan el conocimiento del profesor sobre medidas de dispersión.

Batanero et al. (1997) analizaron las respuestas de 273 futuros profesores de educación primaria a un cuestionario sobre la media, encontrando falta de comprensión del algoritmo de cálculo, de la relación entre media, mediana y moda y del efecto de los valores atípicos sobre estos estadísticos. Resultados similares fueron obtenidos por Estrada, Batanero y Fortuny (2004) en un estudio con 367 futuros profesores de educación primaria.

Groth y Bergner (2006) analizaron la comprensión de 46 futuros profesores de educación primaria sobre la media, mediana y moda, clasificando sus razonamientos según su nivel de comprensión de estos conceptos, desde una comprensión intuitiva del algoritmo de cálculo (sin ser capaz de explicar correctamente las diferencias entre estos estadísticos), hasta un nivel superior (sólo 3 participantes), donde se es capaz de elegir el promedio más representativo para una distribución de datos. Otras investigaciones (Ortiz y Font, 2014; Ruiz, Arteaga y Batanero, 2009) han mostrado la dificultad de los futuros profesores de educación primaria para utilizar las medidas de posición central cuando comparan distribuciones.

Jacobbe (2008) entrevistó a tres profesores de educación primaria en ejercicio, pidiéndoles identificar las situaciones en que la mediana o moda son más representativas en un conjunto de datos. Los profesores no fueron 
capaces de dar una regla general y tuvieron grandes dificultades para decidir cuál promedio era más representativo en ejemplos dados.

Hammerman y Rubin (2004) realizaron un estudio con profesores de educación secundaria, mientras participaban en un curso cuyo objetivo era aprender el uso de un software estadístico para analizar datos. Sus resultados muestran que no todos los profesores utilizaron las medidas de posición central para comparar las distribuciones, aunque el software les proporcionó estas medidas; por el contrario, se centraron preferentemente en comparar las frecuencias en algunos intervalos de las gráficas de las distribuciones. En resumen, los estudios reseñados indican que los futuros profesores de educación primaria tienen numerosas dificultades de comprensión de las medidas de posición central, y algunas de ellas son compartidas por los profesores de educación secundaria.

En relación a la idea de dispersión, Makar y Confrey (2005) analizaron la forma en que 22 futuros profesores de Educación Secundaria verbalizan sus ideas sobre la dispersión al comparar dos distribuciones de datos. Encontraron un vocabulario muy rico, pero poco uso de expresiones estándares y apenas mención a la desviación típica.

Silva y Coutinho (2008) investigaron el razonamiento de 9 profesores de Educación Secundaria en ejercicio sobre la dispersión, sobre una distribución de datos de edades que ellos mismos habían recogido y a partir de la cuál crearon un histograma. Las autoras clasificaron los razonamientos sobre la dispersión en varios niveles, desde uno inicial, donde se calcula la desviación típica sin comprender su significado, hasta el más avanzado (no alcanzado por ninguno de los participantes), que consiste en comprender que en los intervalos formados, sumando y restando k veces la desviación típica a la media, se puede encontrar una cierta proporción de datos. La mayoría de los participantes se clasificaron en el segundo nivel, donde se comprende la desviación típica como medida de las diferencias entre valores o medida de homogeneidad de los datos.

La interpretación de resultados estadísticos es enfatizada, como objetivo de aprendizaje en las orientaciones curriculares. Sin embargo, fue un aspecto difícil en la investigación de Arteaga (2011) con 209 futuros profesores de educación primaria. Al trabajar con un proyecto estadístico, aunque la mayoría elaboraron gráficos y resúmenes estadísticos, fueron pocos los que llegaron a responder a la pregunta propuesta en el proyecto, que requería la interpretación de los mismos. Incluso los que alcanzaron un nivel alto en la lectura de gráficos, obteniendo los promedios o medidas de dispersión, se 
limitaron a presentar sus valores sin interpretar su significado en el contexto del problema.

Nuestro trabajo completa los anteriores, en dos puntos: En primer lugar, al analizar la interpretación del valor de diferentes resúmenes estadísticos, como parte de un proyecto estadístico en una muestra de futuros profesores de secundaria; además, se evalúa la capacidad de elegir un promedio representativo, que también ha sido una tarea difícil de resolver para futuros profesores de educación primaria (Arteaga, 2011; Jacobbe, 2008).

\section{Metodología}

El estudio se desarrolló con dos grupos de estudiantes del Máster de Formación del Profesorado de Educación Secundaria y Bachillerato (en total 65 estudiantes). Fueron informados de la finalidad del estudio, y cedieron sus datos voluntariamente mostrándose muy interesados por colaborar. En la Tabla 1 se resume información de su titulación de procedencia, formación didáctica y experiencia docente. Agradecemos su esfuerzo y el cuidado con que completaron las diferentes actividades.

Tabla 2

Experiencia didáctica de los participantes (\% respecto a la muestra)

\begin{tabular}{lcccc}
\hline & \multicolumn{2}{c}{ Experiencia docente } & Asignaturas de didáctica cursadas \\
\hline Titulación & $\mathrm{Si}$ & No & $\mathrm{Si}$ & No \\
\hline Matemáticas & 22 & 12 & 11 & 23 \\
Estadística & 2 & & 1 & 1 \\
Ingeniería & 11 & 8 & 1 & 18 \\
Otros & 2 & 8 & & 10 \\
\hline Total & 37 & 28 & 13 & 52 \\
\hline
\end{tabular}

Las tareas propuestas se presentan en la Figura 1 y forman parte de un taller formativo sobre enseñanza de la estadística basada en proyectos. El objetivo del taller fue estudiar la variable "Esperanza de vida al nacer", utilizando datos reales sobre la Esperanza de vida al nacer en un conjunto de 194 países. Los datos fueron tomados del servidor de las Naciones Unidas sobre el Índice de desarrollo humano ${ }^{1}$. El taller comenzó analizando con los futuros profesores el significado de la variable Esperanza de vida al nacer y su distribución en el conjunto de datos, para lo cual se construyeron un 
histograma de la distribución, un diagrama de frecuencias acumulados, un gráfico de la caja y bigotes y una tabla con estadísticos resumen. Las actividades analizadas en este trabajo se presentan en la Figura 1.

1. Esperanza de vida. Hemos calculado algunos estadísticos de la Esperanza de vida al nacer en una muestra de 194 países en el año 2009 (Datos de las Naciones Unidas). Interpreta estos estadísticos, que se presentan en la tabla adjunta, e indica qué información proporcionan sobre la distribución de la Esperanza de vida al nacer en el conjunto de países.

2. Teniendo en cuenta la tabla de estadísticos que se muestra y el gráfico de caja y bigotes de la Esperanza de vida al nacer ¿Cuál de los promedios (media, mediana y moda) representaría mejor esta variable? ¿Por qué?

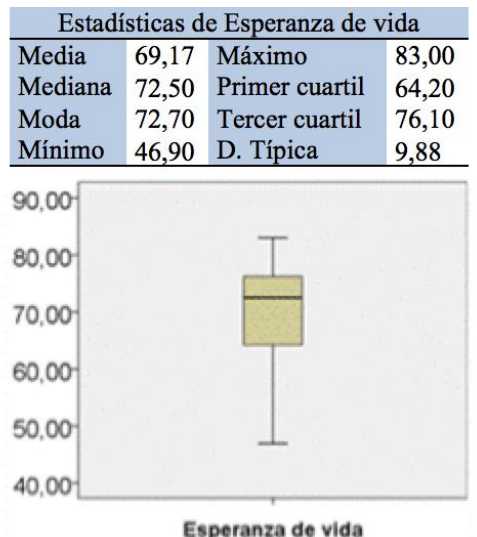

Figura 1. Tareas propuestas

En la primera tarea se pide a los participantes del estudio interpretar los estadísticos de tendencia central, posición y dispersión de la Esperanza de vida. No se les pide interpretarlos todos, sino que elijan alguno de ellos e indiquen el significado de los mismos respecto a la distribución de esperanza de vida. En la segunda tarea, utilizando la tabla anterior y el gráfico de caja y bigotes, se pide elegir el promedio más representativo de la Esperanza de vida, justificando la elección. Puesto que la distribución de esta variable es asimétrica, la mediana o moda (que además, tienen valores cercanos) serían preferibles a la media, como representante de esta variable.

El formador de profesores presentó las actividades, indicando que su fin era promover la reflexión sobre las diferentes medidas estadísticas que pueden usarse en un estudio, y sobre el significado del término "representativo". Indicó que a menudo se necesita trabajar con valores aproximados, como medio de combinación de análisis parciales, para obtenerse con ello índices globales. También aclaró que los valores de la Esperanza de vida son datos agregados (pues son medias ponderadas, no valores de individuos), lo cual tiene repercusión en la interpretación de los estadísticos dados en la tabla. 
Finaliza la presentación sugiriendo que esta actividad podría utilizarse en las aulas de secundaria y bachillerato para dar significado a la noción de media ponderada, concepto difícil para los estudiantes (Batanero, 2000). Igualmente recuerda que algunos estudiantes podrían tener dificultad en comprender el significado de la mediana, respecto a la cual se han descrito numerosos conflictos semióticos (Mayén, Díaz y Batanero, 2009).

\section{Resultados y Discusión}

\section{Interpretación de Estadísticos}

Todos los participantes interpretaron al menos uno de los estadísticos dados en la Tarea 1, siendo los resultados mejores que los obtenidos en las investigaciones con futuros profesores de educación primaria de Arteaga (2011).

Lo más frecuente fue interpretar dos o tres estadísticos diferentes $(24,6 \%$ y $21,5 \%$ de futuros profesores, respectivamente), aunque en algunos casos se llegó a interpretar seis estadísticos (10,8\%). En general, como podemos observar en la Figura 2, los futuros profesores interpretan los estadísticos sin error (56,9\% realizan 0 interpretaciones incorrectas), aunque el 30,8\% comete un error y unos pocos casos cometen más de uno.

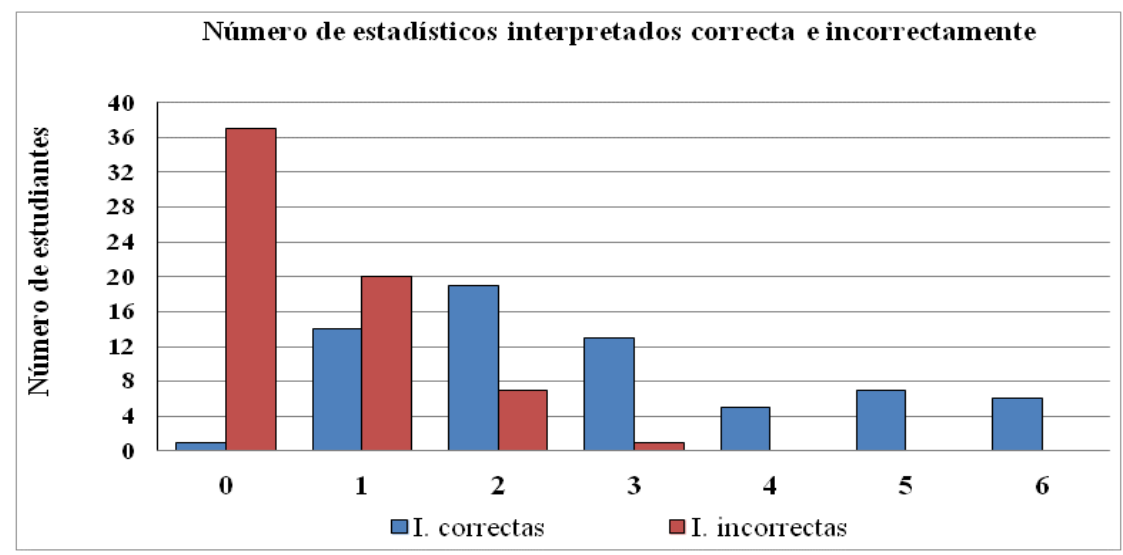

Figura 2. Número de estadísticos interpretados correcta e incorrectamente 
Las respuestas en la tarea se analizaron en función del estadístico interpretado, el grado de corrección y su adecuación al contexto de la variable, obteniéndose las siguientes categorías:

Interpreta la moda. Generalmente la interpretación de la moda es correcta. Por ejemplo, se indica que es el valor más usual o más frecuente en el conjunto de datos, utilizando diversos modos de expresar esta idea y contextualizando la variable analizada:

Lo más repetido en cuanto a la esperanza de vida, es decir, la esperanza de vida con mayor predominio es de 72,7 años (MJG).

Encontramos también sujetos que dan una interpretación incorrecta de la moda en el contexto de la variable, pues confunden la Esperanza de vida con la edad de la muerte. Subyace en la respuesta la tendencia a pensar que los valores de Media y Moda coinciden, ya detectada por Cobo (2003) en su estudio con alumnos de secundaria. La Esperanza de vida es un promedio y en un país tendrá en cuenta la edad de muerte de todas las personas que fallecieron ese año en el país. Pero esta media no tiene por qué coincidir con la moda de la distribución:

Podemos saber también gracias a la moda que a los 75 años es cuando más gente se muere (MC).

Interpretan la media. También en este caso la interpretación suele ser correcta. En la mayoría de los casos, los sujetos que interpretan la media adaptan la definición del valor medio al ejemplo analizado e indican que la media de la Esperanza de vida sería la suma de la Esperanza de vida de todos los países dividida por el número de países en la muestra. En otros, como el siguiente ejemplo, se alcanza una mayor complejidad, al conectar media, mediana y moda en la tabla dada, observando que sus valores son próximos en el ejemplo:

Efectivamente la tabla indica que la media es sobre los 70 años, que casi coincide con el valor de la mitad de la distribución y con el valor más frecuente (EGO).

En pocos casos (sólo tres participantes) se da una interpretación incorrecta de este estadístico, pues se confunde la Esperanza de vida (que es en sí un promedio) con la media de la Esperanza de vida. La confusión es explicable, porque se están manejando dos tipos de media en el problema: La variable Esperanza de vida (que es una media en sí misma: la media de la edad de muerte de todos los habitantes del país) y la media de la Esperanza de vida (que sería la media de todas las esperanzas de vida en los diferentes países): 
Aportan exactitud, viendo así que la esperanza de vida es 69 años (MC).

Interpreta la desviación típica. Son pocos los participantes que interpretan el valor de la desviación típica. Los que lo hacen correctamente, comienzan indicando que es una medida de dispersión y suelen interpretar su significado en relación con la distribución de valores alrededor de la media; en otros casos, pueden señalar que mide la variación (como $\mathrm{CM}$ ) o bien la distancia al valor central (AU):

La variación a la media es en torno a 9,88 años (CM).

[...] la desviación típica es bastante alta, casi 10, y esto quiere decir que los datos están dispersos respecto a la media. Es una pena, quiere decir que hay mucha diferencia entre unos países y otros (AU).

Encontramos algunas interpretaciones incorrectas de la desviación típica como el siguiente ejemplo, donde se considera el valor 9,88 como alto, y se asume por ello que los datos no estarían centrados respecto a la media, es decir, que la distribución no sería simétrica:

Que la desviación típica sea 9,88 quiere decir que los datos no están muy centrados en torno a la media que es 69,17 (ChC).

Interpreta los cuartiles o la mediana. Un ejemplo de interpretación correcta es la de MAG, que interpreta el significado del cuartil en su doble enfoque: por un lado indica el porcentaje de países que tienen una Esperanza de vida menor que dicho cuartil y por otro, el porcentaje de las que lo tienen mayor:

La mitad de los países tiene una esperanza de vida de 72'5 años o menos. La otra mitad tiene una E.V. de 72 '5 años o más. El 25\% de los países tiene una E.V. de 64'20 años o menos, el 75\% de los países tiene una E.V. de 64'2 años o más. El 75\% de los países tiene una E.V. de 76'10 años o menos, el $25 \%$ de los países tiene una E.V. de 76'10 años o más (MAG).

Las dos siguientes interpretaciones son incorrectas. En la primera de ellas se considera que todos los países dentro del rango del percentil poseen la misma Esperanza de vida, en lugar de indicar que su Esperanza de vida sería menor a dicho valor. En la segunda, la moda sí se halla en el intervalo de mayor frecuencia, pero la mediana no tiene por qué encontrarse en dicho intervalo, por lo que hay un conflicto al aplicar a la mediana una propiedad de la moda:

El 25\% de los países posee una esperanza de vida de 64'20 años y el $75 \%$ posee $76 ' 10$ años (MM). 
Tiene sentido que la mediana y la moda se hallen en el intervalo que más frecuencias tiene (MRA).

Interpreta el mínimo. La mayoría de interpretaciones de este estadístico se reducen a explicar que, en los países estudiados, la menor Esperanza de vida es de 46,90 años. Aunque parezca trivial, encontramos tres participantes que muestran un razonamiento incorrecto, confundiendo la esperanza de vida con la edad de muerte, mostrando desconocimiento del contexto, pues es sabido que hay niños que mueren en todos los países:

La persona que antes se muere es a los 46,90 años (CM).

Interpreta el máximo. Al igual que ocurría con las interpretaciones del mínimo, los participantes, por lo general, se limitan a interpretar el máximo contextualizando el dato de la tabla con la variable de estudio. También en este caso encontramos interpretaciones erróneas, al confundir el valor de la variable con la edad de la muerte:

$[\ldots]$ y la última que se muere es a los 83 años (CM).

Interpreta el rango. Los participantes que interpretan este estadístico lo hacen, en su mayoría, correctamente, ofreciendo una interpretación del intervalo a partir de los valores máximo y mínimo:

[...] y que se mueven en el intervalo de [46'90,83'00] (MRA).

Finalmente, resaltamos el hecho de que 20 futuros profesores definen o dan el valor de estadísticos sin interpretarlos. Por tanto, en las explicaciones proporcionadas no contextualizan el significado del estadístico al análisis de la distribución de la variable. El siguiente ejemplo muestra que el participante conoce el significado de los estadísticos que aparecen en la tabla, su explicación es correcta, pero no integra el significado en el contexto de la variable:

Media $\rightarrow$ Punto de equilibrio; Mediana $\rightarrow$ Percentil 50; Moda $\rightarrow$

Valor que más se repite; Mínimo $\rightarrow$ Valor más pequeño; Máximo

$\rightarrow$ Valor más grande; Primer cuartil $\rightarrow$ Percentil 25; Tercer cuartil

$\rightarrow$ Percentil 75; Desv. Típica $\rightarrow$ Dispersión con respecto a la media (ME).

Esta categoría es frecuente al interpretar la desviación típica, seguida, en menor medida, de las interpretaciones de la media y los estadísticos de posición. Estas respuestas, excepto en dos participantes, son combinadas con interpretaciones contextualizadas de otros estadísticos de la tabla refiriéndose a la distribución de la variable Esperanza de vida. La situación es preocupante ya que estos futuros profesores muestran dificultad para 
interpretar ciertos estadísticos en un contexto dado, como ocurrió en el estudio de Arteaga (2011) con futuros profesores de educación primaria.

En la Figura 3 se resumen los resultados de las interpretaciones de los futuros profesores a los estadísticos en la tarea, donde encontramos que la mayoría de las interpretaciones son correctas. Lo más frecuente fue interpretar la moda, seguido de una de las medidas de posición central (mediana o cuartiles), y el máximo o el mínimo, así como el rango. Fueron muy pocos los que interpretaron la desviación típica y en este caso el número de interpretaciones incorrectas casi iguala al de las correctas. El total de interpretaciones incorrectas de los futuros profesores es pequeño (17\% de las interpretaciones aportadas). La mayoría de las interpretaciones incorrectas aportadas por los participantes son de la desviación típica y el máximo (40\% y $26 \%$ de las respuestas incorrectas). El resto de estadísticos tienen menos del $21 \%$ de interpretaciones incorrectas.

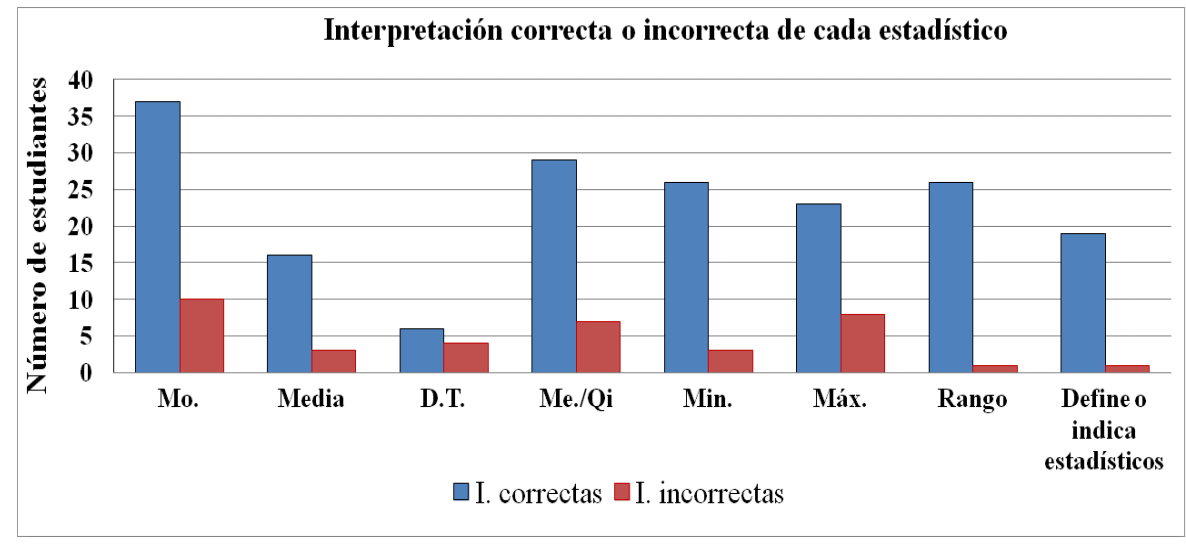

Figura 3. Frecuencia de estadístico interpretado y corrección de la interpretación

\section{Elección del Promedio Representativo}

En el debate de las soluciones aportadas a la tarea anterior, una vez recogidos los datos de la evaluación, se llegó al consenso de que, en este ejemplo, media, mediana y moda tienen un valor muy similar, pero la media es inferior a la mediana o moda, por lo que sería preferible usar la mediana o moda para representar los datos, principalmente por la asimetría de la 
distribución de la variable, como se puede observar en el gráfico de caja y bigotes (ver Figura 1), pues la media es un estadístico no resistente a valores extremos o falta de simetría (Batanero, 2000).

Los resultados en la Tarea 2 se presentan en la Figura 4 y se clasifican atendiendo al estadístico que elige el participante como promedio más representativo, junto al tipo de justificación que realiza de su elección (correcta, incorrecta o no justifica). Observamos que algunos futuros profesores eligen más de un estadístico como promedio representativo o no llegan a decidirse por ninguno (categoría ND), es decir, sugieren que cualquiera de los promedios sería representativo de la distribución. Es pequeño el número de participantes (sólo dos) que no responden a la pregunta.

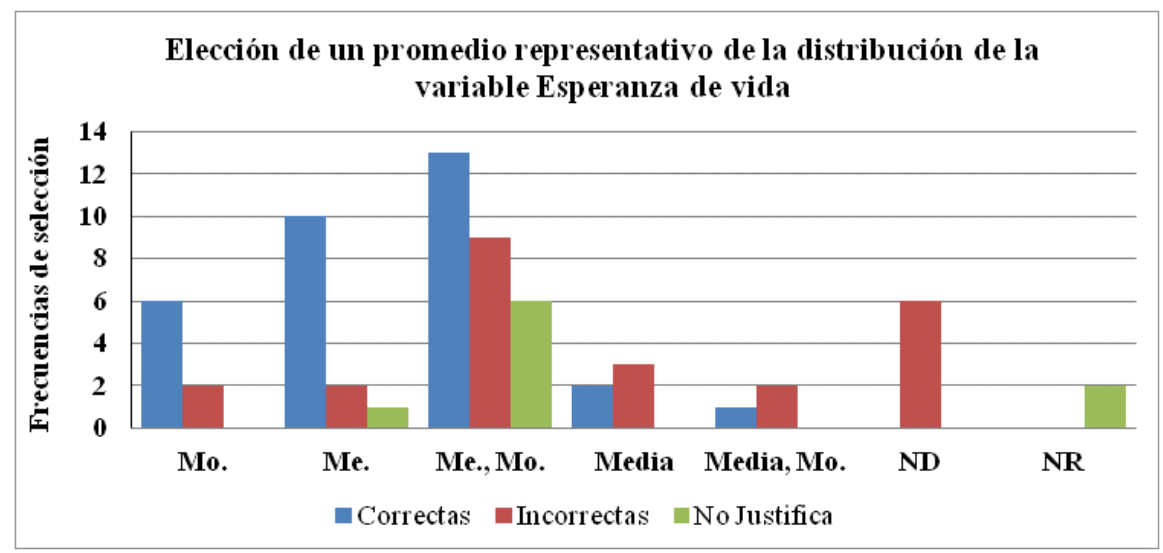

Figura 4. Porcentaje de estudiantes, según promedio elegido y tipo de justificación

A continuación analizamos las respuestas y justificaciones aportadas por los participantes.

Elección de la media. La elección de la media como promedio más representativo en este caso es incorrecta, dada la asimetría de la distribución de la Esperanza de vida, visible en el gráfico de la caja. Entre las justificaciones de esta elección dadas por los participantes, sólo la de CM alude a una propiedad correcta, pues indica que en el cálculo de la media intervienen todos los datos. Las incorrectas se deben principalmente a confusión entre media y mediana, o no comprender bien el significado de la 
desviación típica, como JMV, que asume que la desviación típica indica la distancia de la media al máximo y mínimo:

El dato más representativo es la media porque es el que engloba a todos los países en general (CM).

La media es el valor más representativo porque dista del valor máximo y mínimo 9'88 y la dispersión de los valores máximo y mínimo no es muy grande (JMV).

Elección de la moda. Algunos futuros profesores eligen la moda, lo cuál sería correcto por tener un valor muy cercano a la mediana. La mayoría de las justificaciones de esta elección son correctas; por ejemplo, DG se basa en la frecuencia elevada de países con ese valor de la Esperanza de vida. Encontramos dos justificaciones incorrectas, una de ellas de PG, quien confunde la edad de muerte con el valor de la variable, conflicto descrito al analizar la tarea 1.

Creo que la moda porque es más representativo de la muestra $\rightarrow 55$ países con 75 años de esperanza de vida (DG).

El promedio que mejor representa la esperanza de vida es la moda que es el valor más frecuente de edad en el que se producen más fallecimientos (PG).

Elección de la mediana. Sería una elección correcta y prácticamente todos los futuros profesores que optan por esta elección la justifican correctamente. Suelen indicar que la media se ve afectada por la asimetría de la distribución (EM), o que la mediana separa los países en dos mitades con igual número de efectivos en cada una de ellas. Las pocas justificaciones incorrectas se deben a confusión entre media y mediana, como le ocurre a $\mathrm{AV}$, quien asigna una propiedad incorrecta a la mediana.

La mediana porque la media está demasiado afectada por los valores extremos (EM).

La mediana porque hace una idea general de todos los países, aunque existan excepciones (AV).

Elección de la mediana y la moda como promedio más representativo. El sujeto indica que ambos estadísticos son válidos como representantes de la Esperanza de vida. Muchos optan por estos dos estadísticos, sin justificar su elección. Otros aportan justificaciones correctas, como MAG, que hace referencia a la asimetría y unimodalidad de la distribución. Por su parte, MVC indica que la media se ve muy afectada por los valores extremos:

La media es un mal representante debido a la asimetría de la distribución. Yo tomaría la mediana. Aprovechando que la distribución es unimodal y que hay una gran concentración de datos 
en torno a la moda, también sería un buen representante de la distribución (MAG).

O la mediana o la moda ya que la media es peor porque hace que se compensen los datos (en este caso la media nos hace ver que se vive menos de lo que realmente se vive) la mediana o moda son más representativas (MVC).

A continuación se muestra un ejemplo de justificación incorrecta en esta elección, donde no se tiene información de cuántas personas mueren en el intervalo en torno a la mediana, y además, se razona como si la variable fuese la edad de muerte.

Sería la mediana y la moda, ya que hay mucha más gente que se muere en torno al valor mediano. Pero el valor medio se ve afectado por los valores anómalos y hace que la esperanza de vida se reduzca hasta los 69 años, cuando en realidad es más frecuente morirse a los 75 (EGO).

Elección de la media y la moda. Cuando se eligen estos dos estadísticos; algunos participantes indican que ambos estadísticos tienen valores cercanos y que la mediana no tiene en cuenta el valor de los datos, lo que es incorrecto pues el valor influye en el orden de los mismos (GS). La justificación de AU es correcta, aunque la elección no lo sea, pues las propiedades que describe de la moda y la media son ciertas.

Tengo razones para decir que la que mejor lo representa es la media o la moda. Con la moda sabré cual es la esperanza de vida más común y así podré saber cuál es la esperanza más normal. No obstante no sabré qué pasa con "todo" el mundo. Con la media tendré un poco más de información, si la esperanza media es 69 , me da más información que la moda, aunque con esta no sabré cual esperanza será más común. Me quedo con las dos (AU).

Pienso que serían más acertados tanto la media como la moda, no así la mediana, ya que esta se limita a separar observaciones sin tener en cuenta los valores. Porque son los valores que nos marcan los porcentajes que más se presentan (GS).

No se elige ningún promedio como más representativo. Algunos participantes no eligen ningún promedio, aunque sí responden a la tarea. Las justificaciones que aportan son todas incorrectas, como por ejemplo AJD, que indica que un único resumen estadístico es insuficiente para representar a la variable:

Yo creo que ninguno es representativo por sí solo, habría que darlos junto más medidas (AJD). 
En la Figura 5 comparamos las justificaciones dadas según el promedio elegido (que puede ser más de uno). No se ha diferenciado entre no responder (dos participantes) y responder sin elegir (categoría ND) por lo que estas respuestas se unen en una nueva categoría denotada por NE (No elige). Todos los que indican que no se puede decidir qué promedio es más representativo (12\%) justifican incorrectamente esta decisión. Concluimos que la mayoría poseen dificultad en integrar el significado de estos estadísticos en una decisión, aunque los resultados expuestos en la sección anterior mostraron que todos interpretaron al menos uno de ellos.

Tanto en la Figura 4 como en la 5 observamos que la mayoría de los futuros profesores realizaron una elección correcta, aportando justificaciones adecuadas. Valoramos positivamente los casos (tres participantes) en que se da una respuesta incorrecta, pero se justifica correctamente, ya que da indicios de que los futuros profesores conocen las propiedades de estos conceptos, aunque muestren dificultad en la elección de uno u otro estadístico, como muestra el siguiente ejemplo:

El dato más representativo es la media porque es el que engloba a todos los países, aunque la moda también es importante (CM)

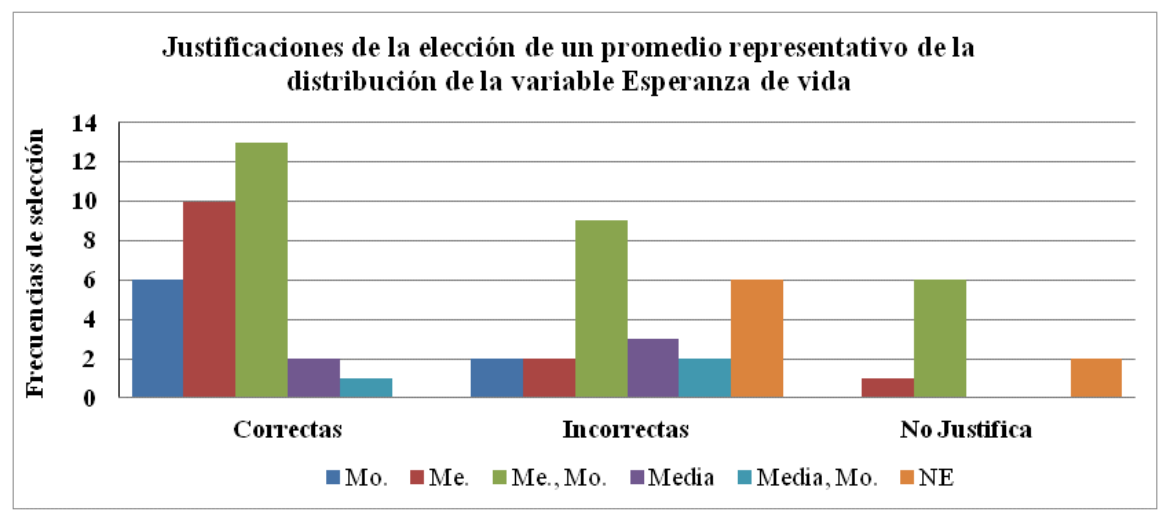

Figura 5. Porcentaje de justificaciones según promedio elegido

Observamos que los futuros profesores que indican conjuntamente la mediana y la moda son los que más justificaciones incorrectas aportan, o bien, no justifican su indecisión. Al comparar los tres promedios, 
paradójicamente, los argumentos correctos aparecen con menor frecuencia en la media, con un alto porcentaje de justificaciones incorrectas $(60 \%)$.

\section{Discusión e Indicaciones para la Formación de Profesores}

La investigación desarrollada proporciona nuevos resultados sobre el conocimiento matemático común de los resúmenes estadísticos en una muestra de futuros profesores de educación secundaria y bachillerato, complementando, de este modo, los antecedentes sobre el tema.

A pesar del alto índice de respuestas correctas, encontramos justificaciones incorrectas en las respuestas a las tareas propuestas $(17 \%$ del total de interpretaciones realizadas por los profesores y $37 \%$ de elecciones del promedio), principalmente debidas a no contextualizar la variable de estudio, que no mide valores de individuos, sino datos agregados (medias ponderadas). Aunque se discutió con los estudiantes su significado, algunos no llegaron a comprenderlo, indicando dificultades con el concepto de media ponderada, ya indicadas por Cobo (2003). También algunos participantes tuvieron dificultad en elegir un promedio representativo, en consonancia con el estudio de Jacobbe (2008).

Los resultados muestran que algunos participantes no comprenden significativamente los estadísticos de valor central y muestran dificultad en integrar el contexto de la variable al significado que aporta un estadístico sobre su distribución (31\% de futuros profesores), aunque la proporción es menor de la encontrada en la investigación de Arteaga (2011) con futuros profesores de primaria. Este autor sugiere que el fallo se produce en el último paso en el proceso de modelización matemática, pues los participantes, aunque son capaces de realizar un trabajo matemático para resolver un problema de investigación, no traducen los resultados de este trabajo a la realidad modelizada.

Estos resultados son preocupantes, pues se espera que el futuro profesor desarrolle en sus estudiantes la capacidad de interpretar resultados de estadísticos, ya que en los diseños curriculares se insiste en ello. Así, por ejemplo, uno de los estándares de aprendizaje evaluables para el cuarto curso de la Educación Secundaria Obligatoria, tanto en la modalidad orientada a la enseñanza académica como en la aplicada (MECD, 2015, p. 398 y p. 407) es: "Interpreta un estudio estadístico a partir de situaciones concretas cercanas al alumno". Por tanto, es necesario que un futuro profesor sea capaz 
de contextualizar la información resumida que le ofrece cada estadístico, para ayudar al alumno a adquirir esta capacidad de interpretación.

Encontramos también dos futuros profesores que no resuelven la segunda tarea. Uno de ellos manifiesta no saber leer el gráfico de caja y bigotes, contenido del currículo para el tercer curso de la Educación Secundaria Obligatoria (ver Tabla 1). El otro indica que no recuerda el significado de la mediana y la moda, que son contenidos de educación primaria. Fue también visible la dificultad de interpretación de la desviación típica.

Las dificultades encontradas, no obstante, fueron menores que las mostradas en otras investigaciones realizadas con profesores de educación primaria, como la de Jacobbe (2008). Algunas de las dificultades observadas han sido las siguientes:

- Confusión entre promedio y valor de la variable, ya mostrado en la investigación de Cobo (2003).

- Falta de criterio para elegir un promedio representativo, llegando a elegir automáticamente la media, incluso en distribuciones asimétricas. Esta dificultad también es observada por Jacobbe (2008) en la identificación de situaciones como la propuesta en nuestra experiencia. Un efecto similar fue observado en Batanero et al. (1997) al no considerar los valores atípicos en el análisis de los estadísticos, así como en Estrada, Batanero y Fortuny (2004). Esta ausencia de criterio se manifiesta en el alto porcentaje de justificaciones incorrectas que aportan los participantes así como la ausencia de justificación a su indecisión.

- Interpretar la desviación típica como un estadístico que mide la asimetría o valorar inadecuadamente el valor de una desviación típica. Makar y Confrey (2005) mostraron que, aunque los futuros profesores de su estudio eran capaces de usar un lenguaje apropiado, sus interpretaciones eran poco profundas; en nuestro estudio, además, una gran proporción de ellas contienen errores (un $40 \%$ de sus interpretaciones eran incorrectas). Resultados similares se encuentran en Silva y Coutinho (2008).

- Interpretación incorrecta del gráfico de la caja, que también aparece en Pfannkuch (2006).

- Dificultad al interpretar los estadísticos en el contexto de la variable, ya que un alto porcentaje de participantes conocen el significado de un estadístico pero no integran el contexto de la variable para 
interpretar su distribución, también mostrado en la investigación de Arteaga (2011).

Concluimos, que es importante completar la formación de los futuros profesores de Educación Secundaria Obligatoria y Bachillerato sobre los resúmenes estadísticos, con talleres y actividades similares a las descritas en este trabajo. Las actividades fueron útiles para desarrollar el conocimiento estadístico en los participantes, además de evaluar sus conocimientos iniciales. El taller resultó interesante a los futuros profesores, que pudieron razonar sobre una variable relevante en la sociedad actual. Al trabajar con fuentes internacionales aumentó el interés de los participantes y su percepción de la utilidad de la estadística. A la vez, la discusión posterior de las soluciones permitió incrementar su conocimiento didáctico sobre el tema. Este trabajo puede completarse con otras propuestas de enseñanza encaminadas al desarrollo del conocimiento estadístico tanto en alumnos como en futuros profesores, como las descritas por Batanero y Díaz (2011). Los proyectos que presentan las autoras se basan en las recomendaciones actuales sobre metodología de enseñanza de la estadística y se conciben para trabajar varios contenidos a la vez, promoviendo el razonamiento estadístico, ya que la estadística se presenta como herramienta en la toma de decisiones, cobrando gran importancia las actividades de interpretación más que de cálculo rutinario. Además las autoras incluyen información sobre la didáctica del tema que permite reforzar el conocimiento didáctico del profesor. Otra propuesta complementaria es descrita por Arteaga (2011), quien propone el trabajo con un proyecto estadístico y posterior discusión de sus características didácticas como medio de aumentar el conocimiento sobre los gráficos y resúmenes estadísticos en los futuros profesores. Igualmente Silva y Coutinho (2008) describen actividades dirigidas a la formación de profesores sobre las medidas de dispersión.

\section{Agradecimientos}

Proyecto EDU2013-41141-P (MEC) y Grupo FQM126 (Junta de Andalucía).

\section{Notas}

${ }^{1}$ Índice de desarrollo humano (Servidor de las Naciones Unidas): http://hdr.undp.org/es/data 
154 María M. Gea et al. - Interpretación de Resúmenes Estadísticos

\section{Bibliografía}

Adler, J. \& Venkat, H. (2014). Mathematical knowledge for teaching. En S. Lerman (Ed,), Encyclopedia of Mathematics Education (pp. 385388). Dordrecht: Springer.

Arteaga, P. (2011). Evaluación de conocimientos sobre gráficos estadísticos y conocimientos didácticos de futuros profesores. Tesis Doctoral. Universidad de Granada. Granada, España.

Ball, D. L., Lubienski, S. T. \& Mewborn, D. S. (2001). Research on teaching mathematics: The unsolved problem of teachers' mathematical knowledge. En V. Richardson (Ed.), Handbook of research on teaching (pp. 433-456). Washington, DC: American Educational Research Association.

Batanero, C. (2000). Significado y comprensión de las medidas de tendencia central. UNO, 25, 41-58.

Batanero, C. y Díaz, C. (2011). Estadística con proyectos. Granada: Departamento de Didáctica de la Matemática.

Batanero, C., Godino, J. \& Navas, F. (1997). Concepciones de maestros de primaria en formción sobre promedios. En H. Salmerón (Ed.), Actas de las VII Jornadas LOGSE: Evaluación Educativa (pp. 310-340). Granada: Universidad de Granada.

Blömeke, S., Hsieh, F. J., Kaiser, G. \& Schmidt, W. (2014). International perspectives on teacher knowledge, beliefs and opportunities to learn. Dordrecht: Springer.

Canada, D. (2004). Preservice teachers' understanding of variation. Tesis doctoral. Portland State University, Portland, Oregon (USA).

Cobo, B. (2003). Significado de las medidas de posición central para los estudiantes de secundaria. Tesis doctoral. Universidad de Granada, Granada, España.

Estrada, A., Batanero, C. \& Fortuny, J. M. (2004). Un estudio sobre conocimientos de estadística elemental en profesores en formación. Educación Matemática, 16, 89-112.

Even, R. \& Ball, D. (2009). The professional education and development of teachers of mathematics. The 15th ICMI Study. New York: Springer.

Groth, R. E. \& Bergner, J. A. (2006). Preservice elementary teachers' conceptual and procedural knowledge of mean, median, and mode. Mathematical Thinking and Learning, 8, 37-63. doi: 


\subsection{7/s15327833mt10801_3}

Hammerman, J. \& Rubin, A. (2004). Strategies for managing statistical complexity with new software tools. Statistics Education Research Journal, 3(2), 17-41. Disponible en http://www.stat.auckland.ac.nz/serj.

Hill, H. C., Ball, D. L. \& Schilling, S. G. (2008). Unpacking pedagogical content knowledge: Conceptualizing and measuring teachers' topicspecific knowledge of students. Journal for Research in Mathematics Education, 39(4), 372-400.

Jacobbe, T. (2008). Elementary school teachers' understanding of the mean and median. En C. Batanero, G. Burrill, C. Reading \& A. Rossman (Eds.), Joint ICMI/IASE Study: Teaching Statistics in School Mathematics. Challenges for Teaching and Teacher Education. Proceedings of the ICMI Study 18 and 2008 IASE Round Table Conference (pp. 1-8). Monterrey Mexico: ICMI e IASE. Disponible en http://iase-web.org/documents/papers/rt2008/T2P13_Jacobbe.pdf.

Jacobbe, T. \& Carvalho, C. (2011). Teachers' understanding of averages. En C. Batanero, G. Burrill \& C. Reading (Eds.), Teaching Statistics in School Mathematics-Challenges for Teaching and Teacher Education (pp. 199-209). New York: Springer.

Llinares, S. \& Krainer, K. (2006). Mathematics (student) teachers and teacher educators as learners. In A. Gutierrez \& P. Boero (Eds.), Handbook of Research on the Psychology of Mathematics Education (pp. 429-459). Rotherdam / Taipei: Sense Publishers.

Makar, K. \& Confrey, J. (2005). "Variation-talk": Articulating meaning in statistics. Statistics Education Research Journal, 4(1), 27-54.

Disponible en www.stat.auckland.ac.nz/serj.

Mayén, S., Díaz, C. \& Batanero, C. (2009). Conflictos semióticos de estudiantes con el concepto de mediana. Statistics Education Research Journal, 8(2), 74-93. Disponible en http://iaseweb.org/Publications.php.

MEC. (2006). Real Decreto 1513/2006, de 7 de diciembre, por el que se establecen las enseñanzas mínimas de la educación primaria. Madrid: Autor.

MEC. (2007a). Real Decreto 1631/2006, de 29 de diciembre, por el que se establecen las enseñanzas mínimas correspondientes a la Educación Secundaria Obligatoria. Madrid: Autor. 
156 María M. Gea et al. - Interpretación de Resúmenes Estadísticos

MEC. (2007b). Real Decreto 1467/2007, de 2 de noviembre, por el que se establece la estructura del bachillerato y se fijan sus enseñanzas mínimas. Madrid: Autor.

MECD. (2014). Real Decreto 126/2014, de 28 de febrero, por el que se establece el currículo básico de la educación primaria. Madrid: Autor.

MECD. (2015). Real Decreto 1105/2014, de 26 de diciembre, por el que se establece el currículo básico de la Educación Secundaria Obligatoria y del Bachillerato. Madrid: Autor.

Ortiz, J. J. \& Font, V. (2014). Pre-service teachers' common content knowledge regarding the arithmetic mean. REDIMAT, 3(3), 192-219. doi: 10.4471/redimat.2014.51

Pfannkuch, M. (2006). Comparing box plot distributions: A teacher's reasoning. Statistics Education Research Journal, 5(2), 27-45. Disponible en http://iase-web.org/Publications.php.

Ruiz, B., Arteaga, P. \& Batanero, C. (2009). Comparación de distribuciones, ¿Una actividad sencilla para los futuros profesores? Trabajo presentado en el II Encontro de Probabilidade e Estatística na Escola. Universidade do Minho, Braga, Portugal.

Shaughnessy, J. M. (2007). Research on statistics learning and reasoning. En F. K. Lester (Ed.), Second handbook of research on mathematics teaching and learning (pp. 957-1009). Charlotte, NC: Information Age Publishing.

Shulman, L. S. (1986). Those who understand: Knowledge growth in teaching. Educational Researcher, 15(2), 4-14.

Silva, C. \& Coutinho, C. (2008). Reasoning about variation of a univariate distribution: a study with secondary mathematics teachers. En C. Batanero, G. Burrill, C. Reading \& A. Rossman (Eds.), Joint ICMI/IASE Study: Teaching Statistics in School Mathematics. Challenges for Teaching and Teacher Education. Proceedings of the ICMI Study 18 and 2008 IASE Round Table Conference (pp. 1-8).

Monterrey Mexico: ICMI e IASE. Disponible en http://iaseweb.org/documents/papers/rt2008/T2P7_Silva.pdf.

Wallman, K. K. (1993). Enhancing statistical literacy: Enriching our society. Journal of the American Statistical Association, 88(421), 18. doi: 10.1080/01621459.1993.10594283 
María M. Gea es profesora ayudante doctor en la Universidad de Granada, España.

Carmen Batanero es catedrática en la Universidad de Granada, España.

José A. Fernandes es profesor asociado en la Universidade do Minho, Portugal.

Pedro Arteaga es profesor ayudante doctor en la Universidad de Granada, España.

Dirección de Contacto: La correspondencia postal sobre este artículo se tiene que enviar a Carmen Batanero. Dirección postal: Facultad de Ciencias de la Educación, Campus Cartuja, 18071, Granada, España. Email: batanero@ugr.es 